\title{
Thiol-disulphide homeostasis in patients with general anxiety disorder and panic disorder
}

\author{
Esra Kabadayi Sahin ${ }^{1 *}$, Gokcen Turan ${ }^{2 \oplus}$, Salim Neselioglu ${ }^{3 \odot}$, Serdar Suleyman Can $^{4 \odot}$, Murat Ilhan Atagun ${ }^{4}$ \\ ${ }^{1}$ Abant Izzet Baysal University, Izzet Baysal Mental Health Research and Training Hospital, Department of Psychiatry, Bolu - Turkey \\ ${ }^{2}$ Ankara City Hospital, Department of Psychiatry, Ankara - Turkey \\ ${ }^{3}$ Ankara Yildirim Beyazit University Medical School, Department of Biochemistry, Ankara - Turkey \\ ${ }^{4}$ Ankara Yildirim Beyazit University Medical School, Department of Psychiatry, Ankara - Turkey
}

\begin{abstract}
Objective: The aim of this study was to evaluate the dynamic thiol-disulphide homeostasis as a novel oxidative stress parameter in newly diagnosed and medication-naive general anxiety disorder (GAD) and panic disorder (PD) patients.

Method: Medication-naive patients with GAD $(n=21)$ or PD $(n=23)$ and healthy individuals $(n=23)$ were enrolled. Patients were administered a sociodemographic form, Beck Depression Inventory, and Beck Anxiety Inventory. Routine biochemical parameters, native thiol, total thiol, and disulphide parameters were measured in all patients and healthy individuals.

Results: The native thiol levels of the GAD and PD groups were found significantly lower than those of the control group $(p<0.001$ and $p=0.03$, respectively), whereas the disulphide levels and disulphide/native thiol ratios of both patient groups were significantly higher than that of the control group $(p<0.001$ and $p=0.011$, respectively). There was no statistically significant difference between the GAD and PD groups with regard to thiol and disulphide levels.

Conclusion: Oxidative stress is considered to be one of the molecular changes underlying the pathogenesis of GAD and PD. This study showed that dynamic thiol-disulphide homeostasis was disturbed due to an increase in oxidants and a decrease in antioxidants in GAD and PD patients, suggesting that antioxidant replacement interventions might be an option in the treatment of these disorders.
\end{abstract}

Keywords: Generalized anxiety disorder, panic disorder, thiol-disulphide homeostasis

\section{INTRODUCTION}

Anxiety disorders are among the most common psychiatric disorders with a prevalence reaching up to $25 \%$ (1). Generalized anxiety disorder (GAD) is defined as persistent, excessive, and uncontrollable worry about daily life events accompanied by irritability, restlessness, muscle tension, and sleep disturbances, affecting approximately $3-6 \%$ of the general population in any given year (2). Panic disorder (PD) is an anxiety disorder characterized by unexpected, sudden, and repeated attacks of fear accompanied by the physical symptoms of hyperarousal lasting for several minutes. The prevalence of PD is between $1 \%$ and $4 \%$ (3). Although certain biological, genetic, and psychosocial theories have been proposed to explain the underlying causes of anxiety disorders, the etiopathogenesis of $\mathrm{GAD}$ and PD is incompletely understood.

How to cite this article: Kabadayi Sahin E, Turan G, Neselioglu S, Can SS, Atagun MI. Thiol-disulphide homeostasis in patients with general anxiety disorder and panic disorder. Dusunen Adam The Journal of Psychiatry and Neurological Sciences 2019;32:289-294.

Correspondence: Esra Kabadayi Sahin, Abant Izzet Baysal University, Izzet Baysal Mental Health Research and Training Hospital, Alpagut Street, Agaccilar Village, 14030, Bolu, Turkey

Phone: +90 3742752525 E-mail: ekabadayi06@gmail.com

Received: March 19, 2019; Revised: July 08, 2019; Accepted: September 19, 2019 
Oxidative stress is defined as an imbalance between antioxidant and oxidant molecules either caused by overproduction of free radicals, deficiencies in antioxidant molecules, or both. The brain is highly sensitive to oxidative stress due to its large amount of oxygen consumption and its relatively modest antioxidant defenses (4). An increasing number of studies are investigating the relationship between oxidative stress and psychiatric disorders, and oxidative stress mechanisms have been implicated in the pathogenesis of different anxiety disorders (5).

There are different methods used to determine the status of oxidant and antioxidant molecules that can constitute oxidative stress in an organism. Thiols, which contain a sulfhydryl (-SH) group, are one of the most important antioxidant molecules that play a major role in the defense against free radicals. Total plasma thiols consist of protein-bound thiols and low-molecularweight thiols. Albumin contains the greatest amount of protein-bound thiols, but the thiols' major defensive function is mostly thought to be performed by lowmolecular-weight thiols such as glutathione, homocysteine, cysteine, and cysteinglycine (6). Thiols can undergo an oxidation reaction with reactive oxygen species (ROS), reducing them and thereby protecting the organism from oxidative damage. Disulphide bonds (-SS-) are formed as a result of these reactions. However, these newly-formed disulphide bridges are reversible and can be reduced to thiol groups again. Thus, a dynamic thiol-disulphide homeostasis is sustained with this turnover. Disruption of the thiol-disulphide homeostasis is thought to be one of the indicators of oxidative stress; it can be evaluated by a novel automated method developed by Erel and Neselioglu (7).

There is some evidence that thiol-disulphide homeostasis is disrupted in both GAD and PD patients. In a recent study with GAD patients, it was shown that increased disulphide levels may cause an imbalance in thiol-disulphide homeostasis (8). On the other hand, it was reported that decreased native and total thiol levels in PD patients can result in an impaired thiol-disulphide balance (9). The aim of this study was to determine the dynamic thiol-disulphide homeostasis using a novel method in medication-naive patients with generalized anxiety disorder (GAD) and panic disorder (PD) and evaluate whether there is a difference between these disorders with regard to thiol and disulphide levels.

\section{METHOD}

The study comprised 120 patients who were admitted to the psychiatry outpatient clinic and diagnosed with either GAD or PD in accordance with the diagnostic criteria of DSM-5. Forty-six patients who previously had a diagnosis of any anxiety disorders or medication use and 30 patients who had a comorbid psychiatric diagnosis (major depression, obsessive-compulsive disorder, social phobia, or minimal cognitive impairment) were excluded. Accordingly, the study was conducted with 44 consecutive outpatients with newly diagnosed GAD $(n=21)$ and PD $(n=23)$, respectively. Age, sex, and body mass index (BMI)matched healthy individuals $(n=23)$ were enrolled as a control group. All participants were between 18 and 65 years of age and medication-naive. Exclusion criteria were the presence of any comorbid chronic disease or psychiatric disorder, a previous diagnosis of anxiety disorder and antidepressant use, or a history of acute infection in the last two months, use of any medication, vitamins, or herbal supplements in the last two months, consumption of tobacco or alcohol, and BMI $>30$. In the clinical assessment, a sociodemographic form, Beck Depression Inventory (BDI), and Beck Anxiety Inventory (BAI) were administered to all the participants. The BDI is a 17 -item self-report scale designed to evaluate emotional, somatic, cognitive and motivational symptoms of depression (10), for which a Turkish validity and reliability study was carried out by Hisli et al. (11). The BAI is a psychometric tool that includes 21 items to assess anxiety symptoms of an individual $(12,13)$.

Approval for the study was granted by the local Ethics Committee, and written informed consent was obtained from all participants prior to study participation.

\section{Measurement of Thiol-Disulphide Homeostasis}

After 8 hours of total fasting, $5 \mathrm{cc}$ venous blood was collected from each participant and transferred into tubes with EDTA (ethylene diamine tetra-acetic acid). Then the blood samples were centrifuged at $1.800 \mathrm{~g}$ for 10 minutes. The serum samples obtained were preserved at $-80^{\circ} \mathrm{C}$ until the biochemical analysis was carried out. For the samples collected from all participants, hemogram and liver, kidney and thyroid function tests were performed and vitamin B12, folate, and ferritin levels were measured. The dynamic thioldisulphide homeostasis was measured using a novel automated measurement method developed by Erel and Neselioglu (7). The native thiol and total thiol contents of the samples were evaluated with a modified Ellman reagent. The disulphide content was obtained 
by dividing the difference between serum total thiol and native thiol levels by two. In addition, disulphide/ native thiol, disulphide/total thiol and native thiol/ total thiol ratios were calculated automatically as percentage (\%).

\section{Statistical Analysis}

Statistical Package for the Social Sciences (SPSS version 22, IBM Inc., Armonk, NY, USA) was used to analyze the study data. Shapiro-Wilks test was run to assess distribution characteristics of the variables. Continuous variables were analyzed with $t$ test or Mann-Whitney $U$ test for comparison of two groups and analysis of variance (ANOVA) or Kruskal-Wallis test for comparing three groups. Levene's test for homogeneity of variances was performed to examine the variances. Post-hoc comparisons were performed with Bonferroni test for homogeneous variances and Tamhane test for non-homogeneous variances. Univariate ANCOVA analyses were performed to investigate the effects of potential confounders (age, gender, BMI, and albumin levels). Means and standard deviations (SD) or median and $25^{\text {th }}-75^{\text {th }}$ percentiles (minimum-maximum) were reported. The chi-square test was used to analyze categorical variables. Pearson's correlation test was used to evaluate correlation between the variables. Statistical significance level was determined as $\mathrm{p}<0.05$.

\section{RESULTS}

A total of 21 GAD patients, 23 PD patients, and 23 healthy controls were included in this study. The demographic features of the patients and the control group are reported in Table 1 . There was no significant difference in age, sex, educational status, and body mass index (BMI) between the three groups ( $\mathrm{p}>0.05)$. The BDI and BAI scores were significantly higher in the patient groups compared to the control group (Table 1). There was no statistically significant difference between the $\mathrm{GAD}$ and $\mathrm{PD}$ groups with regard to BDI and BAI scores $(\mathrm{p}=0.864, \mathrm{p}=1.0$, respectively). There was no statistically

Table 1: Sociodemographic features of the patient and control groups

\begin{tabular}{lcccccc} 
Characteristic & GAD $(\mathbf{n}=\mathbf{2 1})$ & PD $(\mathbf{n}=\mathbf{2 3})$ & Control $(\mathbf{n}=\mathbf{2 3})$ & Eta-Square & $\mathbf{F}, \boldsymbol{\chi}^{\mathbf{2}}$ & $\mathbf{p}$ \\
\hline Age (years) * & $30.67 \pm 6.70$ & $30.09 \pm 8.09$ & $31.48 \pm 5.89$ & 0.01 & 0.232 & 0.794 \\
Gender (Female n, \%)** & $14(66.7 \%)$ & $15(65.2 \%)$ & $17(73.9 \%)$ & & 0.460 & 0.794 \\
Educational status (years)* & $12.62 \pm 3.54$ & $12 \pm 2.97$ & $12.52 \pm 3.91$ & 0.01 & 0.204 & 0.816 \\
BMI (kg/m²) & $23.86 \pm 3.47$ & $23.66 \pm 3.96$ & $24.05 \pm 2.72$ & $<0.01$ & 0.078 & 0.925 \\
BDI score* & $12.57 \pm 4.4$ & $11.48 \pm 5.84$ & $2.09 \pm 2.02$ & 0.55 & 38.998 & $<0.001$ \\
BAl score* & $24.05 \pm 6.73$ & $24.13 \pm 6.15$ & $2.48 \pm 2.29$ & 0.79 & $<0.001$ \\
\hline
\end{tabular}

${ }^{*}$ ANOVA, ${ }^{* *}$ Chi-Square Test. The number of degrees of freedom for the ANOVA was 2.66 and for the Chi-Square test 2. Values are presented as mean \pm standard deviation or $\mathrm{n}$ (percentage) for gender. GAD: Generalized Anxiety Disorder, PD: Panic Disorder, BMI: Body mass index, BDI: Beck Depression Inventory, BAI: Beck Anxiety Inventory. Post-hoc Tamhane Test for BDI: Control<GAD, $\mathrm{p}<0.001$; Control $<\mathrm{PD}, \mathrm{p}<0.001 ; \mathrm{BAl}$ : Control<GAD, $\mathrm{p}<0.001 ;$ Control $<\mathrm{PD}, \mathrm{p}<0.001$.

Table 2: Comparison between the groups with regard to Thiol-Disulphide measures

\begin{tabular}{|c|c|c|c|c|c|c|}
\hline Parameter & GAD $(n=21)$ & PD $(n=23)$ & Control $(n=23)$ & Eta-Square & $F, \chi^{2}$ & $\mathbf{p}$ \\
\hline Native Thiol ( $\mu \mathrm{mol} / \mathrm{L}) *$ & $435.55 \pm 75.72$ & $447.6 \pm 72.49$ & $515.9 \pm 57.08$ & 0.22 & 8.947 & $<0.001$ \\
\hline Total Thiol ( $\mu \mathrm{mol} / \mathrm{L}) *$ & $533.33 \pm 85.33$ & $513.25 \pm 102.43$ & $522.79 \pm 58.49$ & 0.01 & 0.313 & 0.732 \\
\hline Disulphide $(\mu \mathrm{mol} / \mathrm{L}) *$ & $23.29 \pm 10.55$ & $19.95 \pm 8.73$ & $11.20 \pm 7.58$ & 0.23 & 9.297 & $<0.001$ \\
\hline \multicolumn{7}{|l|}{ Pairwise ratios (\%) } \\
\hline Disulphide/Total thiol & $0.28(0.20-0.74)$ & $0.05(0.02-0.47)$ & $0.08(0.00-0.43)$ & & 5.082 & 0.079 \\
\hline$([-\mathbf{S}-\mathbf{S}-] /[-\mathbf{S H}+-\mathbf{S}-\mathbf{S H}])^{* *}$ & $(0.04-0.52)$ & $(0.04-0.26)$ & $(0.02-0.20)$ & & & \\
\hline Disulphide/Native thiol & $0.34(0.20-1.11)$ & $0.06(0.02-0.65)$ & $0.05(0.00-0.41)$ & & 8.194 & 0.017 \\
\hline$([-\mathbf{S}-\mathbf{S}-] /[-\mathbf{S H}])^{* *}$ & $(0.04-0.59)$ & $(0.05-0.34)$ & $(0.20-0.17)$ & & & \\
\hline Native thiol/Total thiol & $0.89(0.48-0.95)$ & $0.91(0.54-1.11)$ & $0.96(0.10-0.93)$ & & 20.906 & $<0.001$ \\
\hline$([-\mathbf{S H}] /[-\mathrm{SH}-+-\mathrm{S}-\mathrm{S}-])^{* *}$ & $(0.74-0.92)$ & $(0.88-0.96)$ & $(0.94-1.00)$ & & & \\
\hline
\end{tabular}

*ANOVA and **Kruskal-Wallis Tests. ANOVA results are reported with F values, Kruskal-Wallis test results are reported with Chi-Square values. The number of degrees of freedom for the ANOVA was 2.66, and for the Kruskal-Wallis test 1. Thiol and disulphide values are presented as mean \pm standard deviation. Pairwise ratios are presented as median (min-max) and (25th-75th percentiles). GAD: Generalized Anxiety Disorder, PD: Panic Disorder Post-hoc Bonferroni test for Native Thiol: $\mathrm{GAD}<$ Control, $\mathrm{p}=0.001$, PD $<$ Control, $\mathrm{p}=0.004$; Disulphide: $\mathrm{GAD}>$ Control, $\mathrm{p}<0.001, \mathrm{PD}>$ Control, $\mathrm{p}=0.011$; Post-hoc Mann-Whitney U test for Disulphide/Native Thiol: $\mathrm{GAD}>$ Control, $\mathrm{p}=0.007$; Native thiol/Total thiol, $\mathrm{GAD}<$ Control, $\mathrm{p}<0.001 ; \mathrm{PD}<$ Control, $\mathrm{p}=0.002$. 


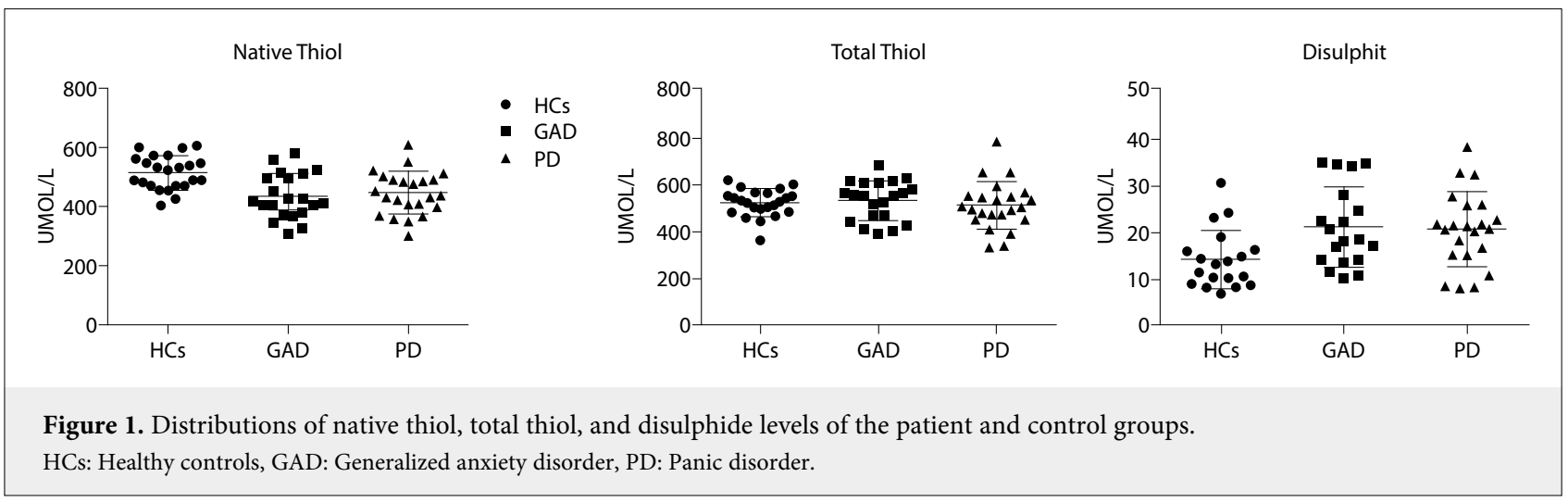

Table 3: Covariate analysis for thiol or disulphide levels

\begin{tabular}{lcccc} 
& Age & Gender & Albumin level & BMI \\
\hline Native Thiol & $\mathrm{F}=0.17, \mathrm{p}=0.681$ & $\mathrm{~F}=0.32, \mathrm{p}=0.573$ & $\mathrm{~F}=0.98, \mathrm{p}=0.325$ & $\mathrm{~F}=0.32, \mathrm{p}=0.574$ \\
Total Thiol & $\mathrm{F}=0.23, \mathrm{p}=0.634$ & $\mathrm{~F}=0.08, \mathrm{p}=0.773$ & $\mathrm{~F}=1.76, \mathrm{p}=0.190$ & $\mathrm{~F}=0.02, \mathrm{p}=0.902$ \\
Disulphide & $\mathrm{F}=3.50, \mathrm{p}=0.066$ & $\mathrm{~F}=1.91, \mathrm{p}=0.172$ & $\mathrm{~F}=1.31, \mathrm{p}=0.257$ & $\mathrm{~F}=2.28, \mathrm{p}=0.136$ \\
\hline
\end{tabular}

Univariate ANCOVA. BMI: Body mass index.

significant difference between the groups with regard to liver, kidney and thyroid function tests, hemogram, ferritin, folate, and vitamin B12 levels ( $p>0.05$ ).

The native thiol, total thiol, and disulphide levels and their pairwise ratios (disulphide/total thiol, disulphide/native thiol, native thiol/total thiol) are presented in Table 2. The native thiol levels and native thiol/total thiol ratios were significantly lower in the GAD and PD groups compared to the control group $(\mathrm{p}=0.001$ and $\mathrm{p}=0.03$, respectively); however, the disulphide levels and disulphide/native thiol ratios of the GAD and PD groups were significantly higher than those of the control group $(\mathrm{p}<0.001$ and $\mathrm{p}=0.011$, respectively). There was no statistically significant difference between the PD and GAD groups in native thiol, disulphide levels and disulphide/total thiol ratio ( $\mathrm{p}=0.933, \mathrm{p}=0.665$, and $\mathrm{p}=0.079$, respectively). There was no statistically significant difference in total thiol levels between the three groups ( $p>0.05)$ (Figure 1). Age, gender, albumin levels, and BMI were investigated as covariates in further analysis. Albumin levels, age, $\mathrm{BMI}$, and gender were all covariates of thiol-disulphide levels ( $p>0.05$ for all, Table 3). Native thiol levels were significantly higher in females than in males in the control group. On the other hand, native thiol levels were found to be significantly higher in males than in females in the PD group ( $\mathrm{p}=0.007)$. Age, education, clinical evaluation scale scores, and all available biochemical variables were controlled for correlations with total thiol, native thiol, and disulphide levels. Total thiol levels and BDI scores had a significant correlation in the PD group (rho=-0.58, p=0.007). No other significant correlation was detected between thioldisulphide levels and clinical or other biochemical variables $(\mathrm{p}>0.05)$.

\section{DISCUSSION}

One of the main findings of this study was that in untreated GAD and PD groups, the native thiol levels and native thiol/total thiol ratios were significantly lower compared to the control group; additionally, disulphide levels and disulphide/native thiol ratios were significantly higher. On the other hand, there was no statistically significant difference between GAD and PD groups with regard to the native thiol and disulphide levels. To our knowledge, this is the first study to compare the oxidative stress status of GAD and PD patients.

Although GAD and PD belong to the same group of disorders and share some common distress symptoms, they differ both with regard to their clinical appearance and their etiopathology. While PD is associated with paroxysmal anxiety episodes, GAD is often characterized by a constant state of anxiety. In addition to environmental differences in etiology, genetic inheritance and familial clustering have been shown to be stronger in PD than in other anxiety disorders (14). Studies in brain imaging and neurotransmitter systems also reveal some differences for both disorders (15). Different studies examined the effects of oxidative stress on GAD and PD separately, 
and the results show certain similarities as well as differences for these disorders.

This study found that the level of native thiol was decreased and the disulphide level was increased in patients with GAD, indicating that thiol-disulphide homeostasis disruption might be due to both decreased levels of antioxidants and increased levels of oxidants. Similar to our results, studies conducted with GAD patients generally observe an increase in oxidant molecules and a decrease in antioxidants in these patients. Different studies on the oxidative status of GAD patients reported reduced total antioxidant levels and elevated total oxidant levels, lipid peroxidation, and oxidative stress index (16-19). A recent study found impaired thiol-disulphide homeostasis in GAD patients. However, that study revealed an increased disulphide level with no difference in the thiol level (8).

Along similar lines, our study also showed decreased native thiol and increased disulphide levels and a disrupted thiol-disulphide balance in PD patients. Previous studies evaluating the oxidative status in PD patients had different results. A recent study also found the thiol-disulphide homeostasis to be impaired in PD patients. However, while it was emphasized that thiol levels were decreased, there were no reported changes in disulphide levels (9). Some studies investigating different oxidative stress markers found that the oxidant enzymes levels (malondialdehyde [MDA], superoxide dismutase [SOD], GSH-Px) increased, and total antioxidant capacity decreased in patients with PD $(20,21)$. On the other hand, some studies found no significant changes in antioxidant enzymes in PD patients or increased total antioxidant capacity that might show a reactive increase of antioxidants to protect the organism against free radicals in $\mathrm{PD}(22,23)$.

We did not find any statistically significant difference with regard to thiol and disulphide levels between GAD and PD groups. Even though there are some etiological differences between the two disorders, these findings might be a result of common factors playing a role in the etiopathogenesis of both disorders. Catecholamines are the major neurotransmitters involved in the pathophysiology of anxiety disorders (24). Reportedly, overactivity of the catecholaminergic system can stimulate the production of reactive oxygen and nitrogen species (ROS, RNS), thus causing an oxidative load. To compensate this load, antioxidant molecules are consumed. If an oxidative balance is not achieved, oxidative stress becomes manifest, potentially causing cellular damage by changing protein expression, membrane fluidity, cellular receptor configuration, and signal transduction (25).

When we examined the effect of BDI and BAI scores on thiol and disulphide levels, we found no correlation between the test scores and oxidative stress parameters other than a positive correlation of BDI scores and total thiol levels in PD. Similar to our findings, other studies in the literature did not determine any correlations between anxiety scores and oxidative stress parameters in $\operatorname{GAD}(8,18)$. Additionally, we found no correlation between oxidative stress status and disease severity in PD (22). This result can be interpreted to mean that an impaired oxidative balance may be related to the disorder itself rather than disease severity.

Another finding of this study was the presence of higher native thiol levels in females than in males in the control group. It is reported that gender has an effect on oxidative status, and females generally have a lower oxidative load than males (26). In addition, estrogen is known as the sex hormone with some antioxidant effects, modulating inflammation (27). The average age of the sample group suggests that the majority of the participants involved in the study were in the premenopausal period. Furthermore, high premenopausal estrogen levels may have caused higher native thiol levels in the healthy control group. On the other hand, this advantage for the female gender disappeared in the patient groups. However, native thiol levels are higher in males with PD than in females, but the very small number of males in the sample group may be a confounding factor when interpreting this finding.

Our study has some limitations: It was conducted in a single center with a relatively small sample size. Another limitation was its cross-sectional design. On the other hand, given the fact that these disorders have high psychiatric comorbidity rates, the involvement of only newly diagnosed and medication-naive GAD and PD patients without any comorbid psychiatric conditions may constitute a strength of this study.

To conclude, these findings can be interpreted to indicate that the thiol-disulphide homeostasis is impaired due to a damaged antioxidant system together with increased oxidant load in both GAD and PD. The causal relationship between anxiety disorders and oxidative imbalance is not clear. It is still not known whether oxidative stress causes anxiety disorders or vice versa. Regardless of the underlying mechanism, any type of therapeutic intervention to reduce oxidative stress or provide antioxidant support should be considered in the treatment of these disorders. 


\begin{tabular}{|c|c|c|}
\hline \multicolumn{2}{|c|}{ Contribution Categories } & Author Initials \\
\hline \multirow{3}{*}{ Category 1} & Concept/Design & M.I.A., E.K.S., S.N. \\
\hline & Data acquisition & E.K.S., G.T. \\
\hline & Data analysis/Interpretation & M.I.A., S.S.C. \\
\hline \multirow{2}{*}{ Category 2} & Drafting manuscript & E.K.S., G.T. \\
\hline & Critical revision of manuscript & M.I.A., S.S.C., S.N. \\
\hline Category 3 & Final approval and accountability & E.K.S., G.T., M.I.A., S.S.C., S.N. \\
\hline \multirow{2}{*}{ Other } & Technical or material support & M.I.A., S.N. \\
\hline & Supervision & M.I.A., S.S.C., S.N. \\
\hline
\end{tabular}

Ethics Committee Approval: Approval for the study was granted by the local Ethics Committee.

Informed Consent: Written informed consent was obtained from the patients.

Peer-review: Externally peer-reviewed.

Conflict of Interest: There is no conflict of interest declared by the authors.

Financial Disclosure: The authors declare that this study has received no financial support.

\section{REFERENCES}

1. Remes $\mathrm{O}$, Brayne $\mathrm{C}$, van der Linde R, Lafortune L. A systematic review of reviews on the prevalence of anxiety disorders in adult populations. Brain Behav 2016; 6:e00497. [CrossRef]

2. Lader M. Generalized anxiety disorder: In: Stolerman IP, Price LH (editors). Encyclopedia of Psychopharmacology. Berlin Heidelberg: Springer, 2015; 699-702. [CrossRef]

3. American Psychiatric Association. Diagnostic and Statistical Manual of Mental Disorders Fifth ed. (DSM-5). Washington DC: American Psychiatric Publ., 2013. [CrossRef]

4. Halliwell B. Oxidative stress and neurodegeneration: where are we now? J Neurochem 2006; 97:1634-1658. [CrossRef]

5. Bouayed J, Rammal H, Soulimani R. Oxidative stress and anxiety: relationship and cellular pathways. Oxid Med Cell Longev 2009; 2:63-67. [CrossRef]

6. Turell L, Radi R, Alvarez B. The thiol pool in human plasma: the central contribution of albumin to redox processes. Free Radic Biol Med 2013; 65:244-253. [CrossRef]

7. Erel O, Neselioglu S. A novel and automated assay for thiol/ disulphide homeostasis. Clin Biochem 2014; 47:326-332. [CrossRef]

8. Asoglu M, Kilicaslan F, Beginoglu O, Fedai U, Akil O, Celik H, Buyukaslan H. Thiol/disulphide homeostasis as a new oxidative stress marker in untreated patients with generalized anxiety disorder. Anadolu Psikiyatri Derg 2018; 19:143-149. [CrossRef]

9. Kulaksizoglu B, Kulaksizoglu S. Thiol-Disulfide Homeostasis in Patients with Panic Disorder. Int J Clin Med 2017; 8:34-41.

10. Beck A, Ward CH, Mendelson M, Mock J, Erbaugh J. An inventory for measuring depression. Arch Gen Psychiatry 1961; 4:561-571. [CrossRef]

11. Hisli N. A study on the validity of the Beck Depression Inventory. Turkish Journal of Psychology 1988; 22:118-126 (Turkish).
12. Beck A, Epstein N, Brown G, Steer R. An Inventory for measuring clinical anxiety: psychometric properties. J Consult Clin Psychol 1988; 56:893-897. [CrossRef]

13. Ulusoy M, Sahin NH, Erkmen H. Turkish version of the Beck Anxiety Inventory: psychometric properties. J Cogn Psychother 1998; 12:163-172.

14. Hettema JM, Neale MC, Kendler KS. A review and meta-analysis of the genetic epidemiology of anxiety disorders. Am J Psychiatry 2001; 158:1568-1578. [CrossRef]

15. Graeff FG, Zangrossi H Jr. The dual role of serotonin in defense and the mode of action of antidepressants on generalized anxiety and panic disorders. Cent Nerv Syst Agents Med Chem 2010; 10:207-217. [CrossRef]

16. Emhan A, Selek S, Bayazit H, Fatih Karababa I, Kati M, Aksoy $\mathrm{N}$. Evaluation of oxidative and antioxidative parameters in generalized anxiety disorder. Psychiatry Res 2015; 230:806-810.

17. Ercan AC, Bahceci B, Polat S, Cenker OC, Bahceci I, Koroglu A, Sahin K, Hocaoglu C. Oxidative status and prolidase activities in generalized anxiety disorder. Asian J Psychiatr 2017; 25:118-122.

18. Bulut M, Selek S, Bez Y, Karababa IF, Kaya MC, Gunes M, Emhan A, Aksoy N, Sir A. Reduced PON1 enzymatic activity and increased lipid hydroperoxide levels that point out oxidative stress in generalized anxiety disorder. J Affect Disord 2013; 150: 829-833.

19. Bal N, Acar ST, Yazici A, Yazici K, Tamer L. Altered levels of malondialdehyde and vitamin $\mathrm{E}$ in major depressive disorder and generalized anxiety disorder. Dusunen Adam 2012; 25:206-211.

20. Kuloglu M, Atmaca M, Tezcan E, Ustundag B, Bulut S. Antioxidant enzyme and malondialdehyde levels in patients with panic disorder. Neuropsychobiology 2002; 46:186-189.

21. Gul I, Karlidag R, Cumurcu B, Turkoz Y, Kartalci S, Ozcan AC, Erdemli ME. The effect of agoraphobia on oxidative stress in panic disorder. Psychiatry Investig 2013; 10: 317-325. [CrossRef]

22. Herken H, Akyol O, Yilmaz HR, Tutkun H, Savas HA, Ozen ME, Kalenderoglu A, Gulec M. Nitric oxide, adenosine deaminase, xanthine oxidase and superoxide dismutase in patients with panic disorder: alterations by antidepressant treatment. Hum Psychopharmacol 2006; 21:53-59. [CrossRef]

23. Ersoy MA, Selek S, Celik H, Erel O, Kaya MC, Savas HA, Herken H. Role of oxidative and antioxidative parameters in etiopathogenesis and prognosis of panic disorder. Int J Neurosci 2008; 118:1025-1037. [CrossRef]

24. Cameron OG, Smith CB, Lee MA, Hollingsworth PJ, Hill EM, Curtis G. Adrenergic status in anxiety disorders: Platelet alpha2adrenergic receptor binding, blood pressure, pulse, and plasma catecholamines in panic and generalized anxiety disorders patients and in normal subjects. Biol Psychiatry 1990; 28:3-20.

25. Schieber M, Chandel NS. ROS function in redox signaling and oxidative stress. Curr Biol 2014; 24:R453-R462. [CrossRef]

26. Brunelli E, Domanico F, La Russa D, Pellegrino D. Sex differences in oxidative stress biomarkers. Curr Drug Targets 2014; 15:811-815.

27. Pietraforte D, Straface E, Piscopo P, Vona R, Confaloni A. Sexrelated biomarkers in cardiovascular and neurodegenerative disorders. Ann Ist Super Sanita 2016; 52:230-239. 